\title{
Programa de pósteres
}

P1. Búsqueda y uso de información científica: colaboración del CRAI-Biblioteca de Farmacia en el Trabajo de Fin de Grado de la titulación del Grado de Farmacia en la Universidad de Barcelona

Arenas Ortigosa M, Kleque Garcia M, Manso Santolaria A, Sánchez Monterrubio J

P2. El uso sexista del lenguaje en las webs de facultades y escuelas de Ciencias de la Salud de la UB

Olivé MC, Mentado T, Montané A

P3. Evolución de los resultados sobre la formación transversal de los estudiantes del Grado de Farmacia en la Unidad de Laboratorios Docentes de la Facultad de Farmacia de la Universidad de Barcelona

Rodríguez-Lagunas MJ, Girona V, Martín-Venegas R, Obiols N, Payà M, Piqué B, Fisa R, Prat J, Ferrer R

P4. Evaluación única o continuada. ¿Qué motivos influyen en la elección del estudiante? Rozas MR, Garrido E, Martínez C, Sancho R

P5. Rendimiento académico de los estudiantes del Grado de Farmacia según la vía de acceso a los estudios (CFGS frente a PAAU)

Amat $C$, Marquès A, Miñarro $M$, Pallàs $M$

P6. Psicología y profesión: acercando los mundos académicos y laborales

Corral MJ, Jarne A, Dolado R, Luna MC, Carmona M, Ramírez C, Arch M, Colell M, Adan A, Del Cerro A, Carreras MV, Aparicio S

P7. Alcoholismo: un caso clínico de abordaje multidisciplinar en la Facultad de Farmacia

Cambras T, Baldomà L, Campanera JM, Canudas A, Franch A, Gómez-Catalàn J, March MA, Martin-
Venegas R, Metón I, Modamio P, Moreno JJ, Noé V, Pubill D, Vazquez M, Zulaica E, Rodamilans M

P8. Valoración por parte de los estudiantes de diferentes modalidades de tutoría grupal Rivero M, Celdrán M, Martí E, Ribera N, Villar F

P9. Las buenas prácticas para la promoción de la parentalidad positiva

Molina MC, Balsells A, Casas J, Fuentes M, Fuentes-Peláez N, Mateos A, Mundet A, Pastor C, Ponce C, Urrea A, Vaquero $\mathrm{E}$

P10. Capacitación de los estudiantes de farmacia como agentes educadores de la salud Fuentes M, Prat A, Molina MC, March M

P11. Relevancia de la competencia transversal Capacidad de aprendizaje y responsabilidad en el ámbito de ciencias de la salud

Gracenea M, Halbaut L, Aróztegui M, Costa Ll, Godall M, González-Moreno O, Calpena A, Carmona AM, Garcia Celma MJ, Suñer J

P12. Relevancia de la competencia transversal Compromiso ético en el ámbito de ciencias de la salud

Gracenea M, Halbaut L, Aróztegui M, Costa L, Godall M, López-Tamames E, Fuentes M, Manzanares C, Simón J, Suñé-Negre JM

P13. Elaboración de rúbricas de evaluación de competencias transversales: una integración del EEES en prácticas tuteladas de la Facultad de Farmacia (Universidad de Barcelona)

March M, Aguilar R, Amat C, Cabañas MJ, Caelles N, Canela M, Carbonell C, Casas J, Escubedo E, García I, Gascón MP, Giner P, Jodar R, Lladonosa L, Mangues I, Mas A, Masip M, Miró A, Oller H, Pla L, Simón J, Soy D, Tribó G, Via MA, Zulaica E 
P14. Valoración de elementos transversales de aprendizaje durante el trabajo en equipo de estudiantes de odontología en presentación de seminarios de anatomía

Valdivia-Gandur I, Sanchez-Bizjak R, Carvalho-Lobato P, Tallón-Walton V, Manzanares-Céspedes MC

P15. Uso de la webquest como herramienta para la adquisición de competencias transversales del Grado de Farmacia

Alegret M, Pallàs M

P16. Análisis de la evaluación única y continuada en Biofarmacia y Farmacocinética I

Escribano E, Colom H, Lauroba J, Calpena AC, Boix A, Díez I, Cendrós JM, Fernández F, GarciaSala X, Peraire C

P17. Evaluación del rendimiento académico en diseños de investigación

Bono R, Núñez MI, Suárez M, Arnau J

P18. Nivelación de conocimientos en el tránsito bachillerato-universidad: análisis de los contenidos en microscopía en el Grado de Farmacia Barceló MC, Gómez Garreta A, Ribera MA, Aguilar E, Blanché C

P19. La evaluación continua a través del cuestionario Moodle. Opinión de los estudiantes

Torres B, Vallès A, Canals M, Potau JA

P20. Adquisición de habilidades para la investigación

Cuffí ML, Fernández V, Fernández A, Sánchez S, Ciruela F, Vallano A, Arnau JM, Carbonell L

P21. El estudio de las malformaciones bucodentales y su influencia en el diagnóstico precoz de enfermedades raras

Tallón-Wallton V, Carvalho-Lobato P, Viñals-Iglesias H, Valdivia-Gandur I, Sánchez-Bizjak R, Manzanares-Céspedes MC

P22. Conocimientos sobre salud sexual y reproductiva en estudiantes de Farmacia (Trabajo de Fin de Grado)

Andreu X, Fuentes M, Molina MC

P23. Integración docencia e investigación: automedicación y uso de terapias complementarias en estudiantes universitarios

Puig M, Moreno MC, Falcó A, Garrido E, Solà M, Roca M, Torres P, Úbeda I
P24. Observaciones y valoraciones realizadas por los estudiantes sobre la actividad 'Inspeccionem els laboratoris farmacèutics?'

García Montoya E, Silva C, Pérez Lozano P, Ticó JR, Miñarro M, Suñé Negre JM

P25. Evaluación del curso de Dermofarmacia para la identificación de acciones de mejora de la docencia

Del Pozo A, Viscasillas A, Juve J, Halbaut L

P26. Actividades de aprendizaje de tipo aplicativo en el estudio de casos en grado y posgrado Rodríguez-Lagunas MJ, Castellote C, Castell M, Franch A, Pérez-Cano FJ

P27. Seminarios prácticos mediante el aprendizaje basado en problemas en la enseñanza de la pediatría

Noguera-Julian A, Fortuny C, García J, Pou J

P28. Implementación de actividades de carácter voluntario como herramienta de aprendizaje en grupos de estudiantes numerosos

Martín-Venegas R, Vinardell MP, Martínez V, Moran C, Cambras T, Díez A, Mitjans $M$

P29. Planificar la formación de posgrado respecto a los cuidados del paciente crítico

Moreno MC, Jerez SA, Andreu L

P30. Valoración de la evaluación continuada en la asignatura de Fisiología y Fisiopatología III del Grado de Farmacia

Pelegrí C, Martín-Venagas R, Moreno JJ, Rabanal M, Pérez-Bosque A, Vilaplana J, Ferrer R

P31. Implantación de la asignatura Introducción a la Clínica Podológica en el Grado de Podología

De Planell E, Novel V, Prats B

P32. Aprendizaje de anatomía topográfica maxilofacial mediante análisis de imágenes de disección por planos incluyendo planteamientos clínicos y quirúrgicos para estudiantes de odontología

Valdivia-Gandur I, Sánchez-Bizjak R, Carvalho-Lobato P, Tallón-Walton V, Manzanares-Céspedes $\mathrm{MC}$

P33. Evaluación continuada en Odontología con sistemas automáticos de preguntas y respuestas Berástegui E, Caudevila C 
P34. Conocer a los estudiantes para mejorar la docencia: hábitos de estudio y de ocio de los estudiantes de Farmacia de la UB

Pujol Cubells M

P35. Diseño e implantación de una nueva práctica informática para promover el aprendizaje activo de la asignatura Microbiología II

Farfán M, Marqués AM, Fusté MC

P36. Enfrentarse a la realidad asistencial. Evaluación de una actividad práctica

Isla P, López C, Rigol A, Borras JM, Guanter L, Gil FL, Julve M, Fernández E

P37. Grado de satisfacción con las prácticas de modelos óseos en los estudiantes de Cirugía Podológica

De Planell E, Novel V, Giralt E

P38. Reflexiones de los estudiantes respecto a su aprendizaje en los centros de atención primaria (CAP)

Solà M, Fabrellas N, Pulpón AM, Sancho R, Olivé E

P39. Evaluación del Prácticum en industria del Máster universitario de Investigación, Desarrollo y Control de Medicamentos

García L, Egea MA, Calpena A, González E, Fernández F, Bielsa R, Espina M, Gamisans F, GarcíaCelma MJ, Morral G, Nikolic S, Salvadó MA, Souto E, Valls O, Vega E, Oliva M, Bel E, Boix A, Clarés B, Colom H, Escribano E, Esteve J, Flo A, Garduño ML, Lauroba J

P40. Valoración de la última edición del Prácticum de licenciatura de la Facultad de Psicología: retos para las prácticas en centros del grado Guilera G, Carreras MV

P41. Autoaprendizaje en prácticas de Farmacia Clínica

Martin MJ, Mallandrich M, Abrego G, Gálvez P, Calpena AC, Ruiz MA, Fernández F, Clares B

P42. Aprender con la práctica: control de materias primas según NCF del título de especialista en farmacia industrial y galénica

Carrillo C, Garcia-Montoya E, Fábregas A, Miñarro M, Pérez-Lozano P, Sánchez N, Sarrate R, SuñéNegre JM, Ticó JR
P43. Introducción de la simulación avanzada en el Grado de Enfermería

Sarria JA, Basco L, Bernárdez J, Estrada JM, Hidalgo MA, González A, Fernàndez A, Fabra M

P44. Alta simulación en enfermería. Máster en Enfermería Urgencias Hospitalarias

Sarria JA, Fabra M

P45. Simulación de actividades profesionales en la oficina de farmacia desde la asignatura Gestión y Planificación

Bonet Clols F, Martin Barea MP, Gaspar Garcia MD, Bel Prieto E

P46. Reflexión y análisis sobre la estructura de las prácticas de la Diplomatura de Nutrición $\mathrm{Hu}$ mana y Dietética (NHD) de la Universidad de Barcelona Illán M, Padró L, Palma I

P47. Adaptación interuniversitaria para la evaluación de competencias en laboratorios de ciencia y tecnología

Calpena AC, Mallandrich M, Oliva M, Fernández F, García ML, Garduño ML, Gálvez P, Martín MJ, Clares B

P48. Red en neurociencias iberoamericana (GENIAR). Experiencias en cursos de formación a nivel de posgrado

Camins A, Junyent F, Verdaguer E, Lazarowski A, Pallàs M, Folch J, Auladell C, Beas-Zarate C

P49. La metodología learning by doing en las prácticas de comprimidos de la titulación Especialidad en Farmacia Industrial y Galénica Fábregas A, García-Montoya E, Pérez-Lozano P, Miñarro M, Sarrate R, Carrillo C, Sánchez N, Ticó JR, Suñé-Negre JM

P50. Incorporación de pequeñas secuencias de cine comercial en la enseñanza de la toxicología Piqué E, Gómez-Catalán J, Rodamilans N, Llobet JM, Borrego N, Barrot C, Teixidó E, Boix N

P51. Experiencias de innovación docente: webquest y clase activa en la docencia de Historia de la Farmacia

Esteva de Sagrera J, Carmona Cornet AM 
P52. Nanotecnología farmacéutica: integración de investigación y docencia en una asignatura de máster oficial

Morral-Ruiz G, Salvadó MA, García-Celma MJ

P53. Actividad semipresencial en la asignatura Clínica Odontológica Integrada Infantil (COII) Pascual M, Sànchez M, Brau I, Ustrell JM

P54. Farmacología y toxicología en $I+D+i$ : adquisición de competencias a través de un ejemplo del desarrollo de un fármaco

Llobet JM, Alegret M, Escubedo E, Janer G, Merlos $M$, Pallàs $M$
P55. Implementación del Plan Bolonia (EEES) en una asignatura de medicina fundamentalmente quirúrgica

López-Boado MA, Rull A, Fontdevila J, Valero R, Fita G, Rovira I, Fábregas N, Gomar C

P56. Los sistemas personalizados de dosificación en vídeo como herramienta docente en la farmacia clínica y la atención farmacéutica

Modamio P, Ruiz-Loscertales H, Vilaró L, MiguezDíez E, Braza AJ, Tobaruela G, Lastra CF, Mariño EL 